\title{
Using Fuzzy Logic in High Level Control Functions
}

\author{
S. L. Chiu \\ Rockwell Science Center \\ 1049 Camino Dos Rios, Thousand Oaks, CA 91360, USA \\ Phone: 805-373-4408, FAX: 805-373-4383 \\ Email: slchiu@rsc.rockwell.com
}

\begin{abstract}
Most literature on fuzzy control deals with the use of fuzzy rules to implement low-level control functions, such as nonlinear proportional-integral-derivative (PID) type control. This paper discusses various ways that fuzzy logic can be used in high-level control functions. Specifically, we examine the use of fuzzy logic for supervisory control, for classifying the operating environment, for process modeling and optimization, and for expressing controller performance metrics. The purpose of this paper is to stimulate the use of fuzzy logic to provide new control functions that are outside the domain of traditional control.
\end{abstract}

\section{Keywords}

Fuzzy control, supervisory control, process optimization

\section{INTRODUCTION}

The fuzzy controllers described in most technical literature deal with the set-point regulation problem, where the control objective is to drive a process variable (e.g., motor shaft position, oven temperature) to a commanded set-point. If one reads a paper on fuzzy control, chances are the paper will describe a fuzzy controller with set-point error and error change as its inputs, and the output is an actuator command or a change in actuator command. When used in this way, fuzzy control is not much different from conventional PID (proportional-integral-derivative)

\footnotetext{
The original version of this chapter was revised: The copyright line was incorrect. This has been corrected. The Erratum to this chapter is available at DOI: 10.1007/978-0-387-35390-6_58 
control - it is solving the same set-point regulation problem addressed by PID control and solving them in essentially the same way as PID control, except that fuzzy control provides a nonlinear input/output mapping. Hence, fuzzy control is often viewed as a form of nonlinear PID control, and comparisons of fuzzy control versus conventional PID control abound in literature. Many engineers lose interest in fuzzy control after finding the performance improvement offered by fuzzy PID control cannot offset the increased complexity in computation and controller tuning, or after finding nonlinear PID control can be more efficiently implemented through other mechanisms (e.g., gain scheduling or look-up table) than through fuzzy rules. Conventional PID control is well-established and can satisfy the performance requirements of most set-point regulation problems at minimal cost; there is little incentive to switch from conventional PID control to a more complex, nonlinear form of PID control unless the conventional controller is doing an unsatisfactory job. Hence, the fuzzy PID controller that is ubiquitous in technical literature rarely appears in actual industrial applications; industrial applications of fuzzy control largely use fuzzy logic in high-level control functions.

The misconception in equating fuzzy control with fuzzy PID control has steered many engineers away from exploiting the full potential of fuzzy logic in control applications. The purpose of this paper is to point out the many ways that fuzzy logic can be used beyond fuzzy PID control. In particular, we will examine the use of fuzzy logic in a variety of high-level control functions. The high level control functions discussed here include supervisory control, classification of the operating environment, process modeling and optimization, and evaluation of controller performance.

\section{SUPERVISORY CONTROL}

Fuzzy control in the form of nonlinear PID control has not found much acceptance in industry, because conventional PID control is well entrenched, simpler, low cost, and works satisfactorily for most applications. For the instances where fuzzy logic is applied to set-point regulation, it is typically used in a high-level module that supervises a conventional PID controller. Here we give several examples to illustrate different forms of fuzzy supervisory control.

The temperature controller produced by Yokogawa Electric (Yokogawa 1990) is a good example of how fuzzy logic can be used for supervisory control. Temperature control usually involves processes that have a long time delay; for many processes, it is also imperative that the temperature does not overshoot the desired set-point. However, it is difficult to avoid overshoot when a process has a long time delay, except by using low feedback gains, which results in slow system response. In Yokogawa Electric's temperature controller, fuzzy logic is used to determine artificial set-points that are fed to a conventional PID controller. The control architecture is shown in Figure 1. The PID controller is allowed to have high feedback gains for fast system response. As the fuzzy supervisory module 
detects impending overshoot, it "fools" the PID controller by commanding the PID controller to aim for a temperature value that is somewhat lower than the actual set-point. As the temperature rises to (and overshoots) the artificial set-point, the fuzzy module gradually raises the artificial set-point toward the actual set-point. In this way, the fuzzy supervisory module leads the PID controller along a temperature trajectory that can quickly reach the actual set-point without overshoot.

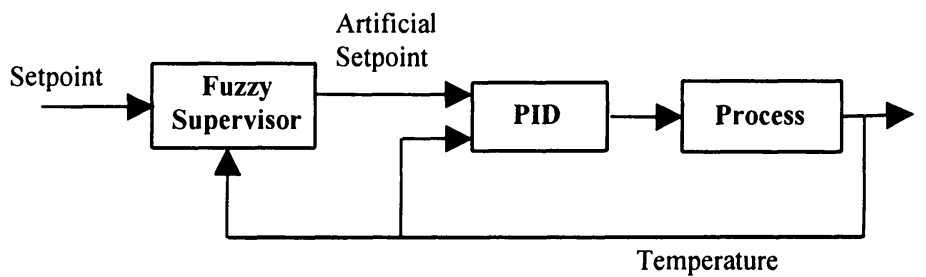

Figure 1 Conventional PID control is assisted by a fuzzy supervisor to prevent overshoot.

In a motion controller produced by Allen-Bradley, fuzzy logic is used to supervise the automatic tuner of a conventional PID controller (Chand 1991). The tuner observes the system response and automatically adjusts the PID controller feedback gains during successive tuning cycles to obtain desired system response characteristics. To facilitate fast convergence to the optimal gain values, and to protect the system against incorrect gain changes, a fuzzy supervisory module scales the output of the tuner (the gain adjustment commands) based on the resultant system performance. The fuzzy module adaptively scales the tuner's output based on the amount of performance improvement after each tuning cycle and the consistency of the performance improvement over the past few tuning cycles. The tuner's output is scaled up if the system performance is consistently improving, and scaled down if the tuner becomes ineffective in further improving performance or if the performance vacillates.

In a steam turbine control application at General Electric, a fuzzy supervisory module is used to combine the output of several conventional PID controllers (Bonissone et al. 1995). The turbine control system employs three PID controllers for regulating the turbine temperature, speed, and stress, respectively. However, there is only one control actuator (the bypass valve) for regulating these three parameters. Therefore, the different, often conflicting actuator commands from the three PID controllers must be resolved into a single command. The fuzzy supervisory module assigns weights to the different PID controller outputs based on the high-level control objective specified by an operator and on the current system state (temperature, speed, and stress). For example, if the objective is to prewarm the turbine as fast as possible, then temperature control would be given higher priority than speed and stress control unless the speed or stress are significantly misbehaving. This control architecture is shown in Figure 2. Fuzzy 
logic has also been used similarly for gain scheduling, where either the outputs or feedback gains of linear controllers designed for different operating conditions are blended based on fuzzy rules (Sugeno and Kang 1986; Gonsalves and Zacharias 1994).

In most process control applications, a human operator must determine the setpoints for numerous PID controllers and periodically adjust the set-points to adapt to changing process conditions. Another type of fuzzy supervisory control involves converting the human operator's knowledge into a set of fuzzy rules, and thus creating a high level controller that automatically determines the set-points for the low-level PID controllers. Froese et al. (1994) describes an example of this type of fuzzy supervisory control applied to slurry treatment.

The capability of a control system can be greatly enhanced by adding a supervisory module to complement conventional control algorithms. The implementation of intelligent supervisory functions is usually straightforward using fuzzy logic. The examples described above provide only a glimpse of the possibilities.

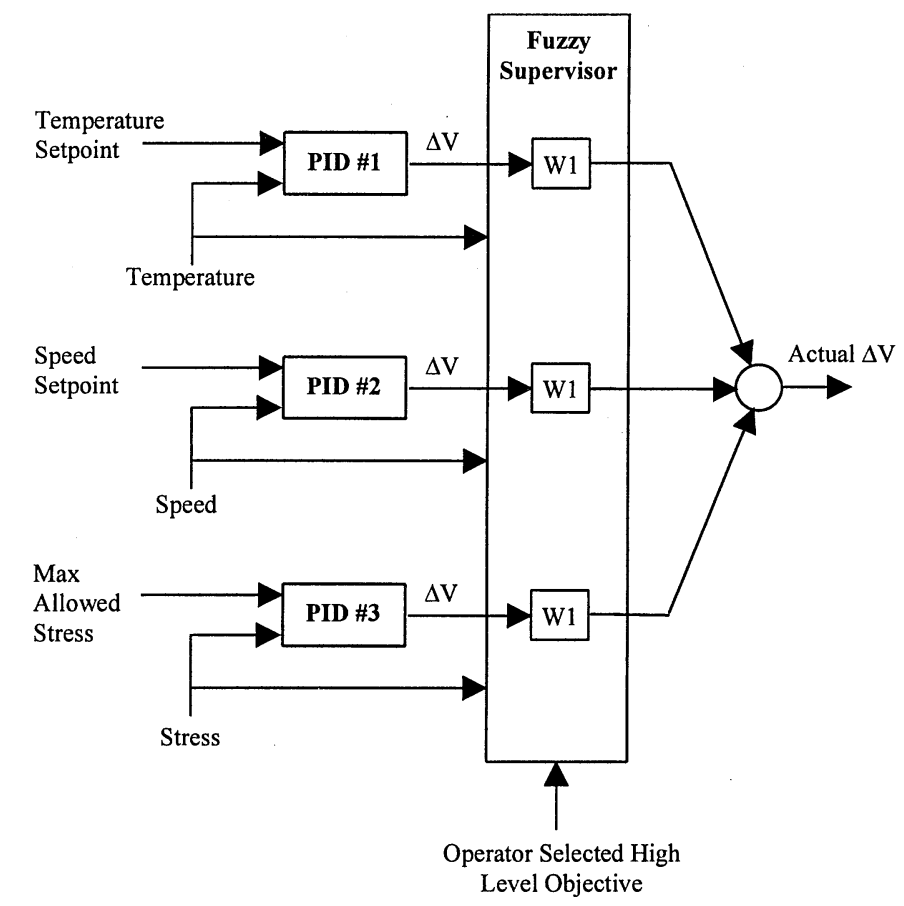

Figure 2 A fuzzy supervisor assigns weights to the output of different PID controllers according to the current system state and the control objective specified by a human operator. 


\section{CLASSIFICATION OF OPERATING ENVIRONMENT}

Typically a simple control law can provide good performance within only a limited range of operating conditions. Thus, many control systems require switching between different control laws as the operating condition changes. Gain scheduling, whereby the controller feedback gains are switched to different values as the plant state moves from one operating region to another, is commonly used in conventional control systems to compensate for the limitations of linear control laws.

For set-point regulation problems, switching between different control laws is usually a straightforward function of the measured plant states (e.g., temperature, speed). However, at higher levels of control, switching between different control laws, or control strategies, is based on high level characterizations of the operating environment (e.g., heating a large mass or a small mass, driving on a highway or city street). Because the high level characteristics of interest are often not directly measurable, the characteristics often need to be inferred from indirect sensor measurements. In such cases, fuzzy logic is extremely useful for encoding the heuristics to infer the characteristics.

An automatic transmission controller developed by Nissan Motors (Takahashi 1991) illustrates the use of fuzzy logic to characterize the operating environment. This automatic transmission controller holds a set of shift patterns that are optimized for different driving environments (e.g., highway driving, mountain driving, city driving), and selects the appropriate shift pattern according to the driving environment. For example, human drivers prefer to maintain a constant gear when driving on a winding road, although the throttle must change constantly. Therefore, driving on a winding road calls for a shift pattern that is less sensitive to throttle change than a shift pattern designed for highway driving. In this automatic transmission controller, fuzzy logic is not used to directly control shifting, but to identify the driving environment so that the appropriate shift pattern can be selected to control shifting.

Because there are no suitable sensors for identifying the driving environment, Nissan engineers used fuzzy logic to infer the driving environment from the driver's accelerator input. For example, the accelerator input tends to be small and constant when driving on a highway, while the accelerator input fluctuates wildly when driving on a winding road (see Figure 3). Fuzzy membership functions were defined to characterize the accelerator input, variance in accelerator input, and vehicle speed that the driver produces in each driving environment (see Figure 4). The controller evaluates the degree to which the accelerator and speed data match the condition associated with each driving environment. Based on the driving environment with the highest degree of match, the appropriate shift pattern is applied to control shifting. 
Driving on Highway

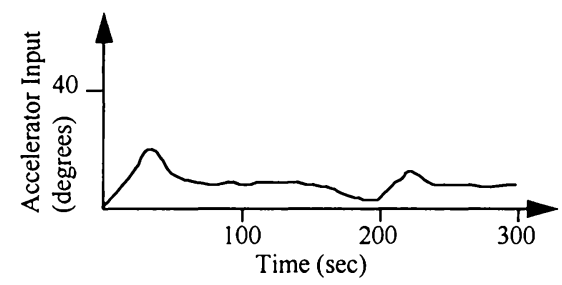

Driving on Winding Road

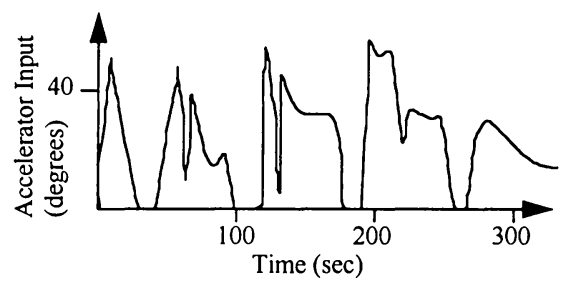

Figure 3 A driver produces distinctive accelerator input behaviors when driving on a highway versus driving on a winding road.
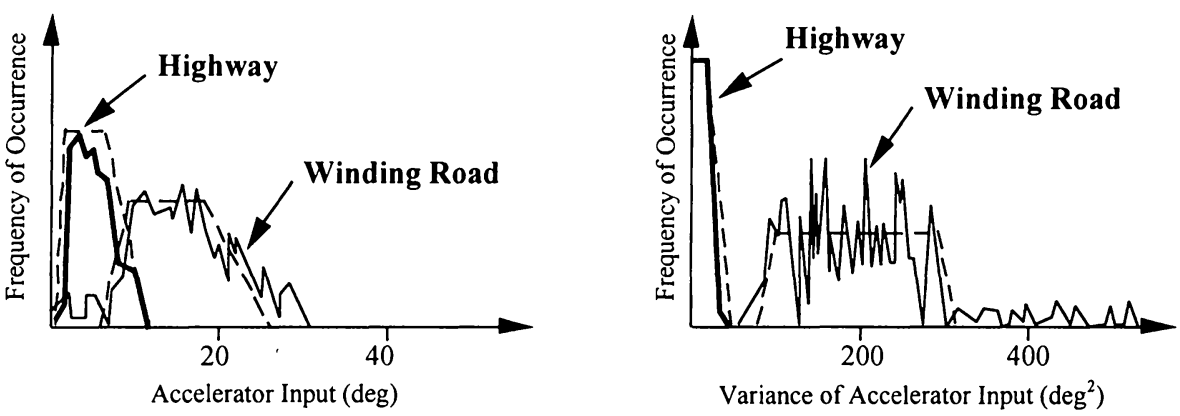

Figure 4 Membership functions characterize the accelerator input and variance in accelerator input produced by the driver in different driving environments.

In addition to using fuzzy logic to infer the operating environment, another interesting aspect of this application is the use of the human driver as the environment sensor. Creatively inferring information from indirect sensors (often sensors that seem unrelated to the characteristic that we want to measure) is at the heart of many successful fuzzy control applications.

The "intelligence" of a controller is dependent on the amount of information available to the controller. When there is a lack of directly measurable information, our tendency is to try to design a robust controller that provides acceptable performance under all variations of the unknown (e.g., an automatic transmission shift pattern that works adequately for all driving environments). Instead of accepting merely adequate performance, we should challenge ourselves to find ways to obtain the information needed for optimal performance. In many cases, the information needed to optimize control choices can be inferred from indirect sensor measurements. Creativity of the system designer in inferring information from indirect sources, coupled with the power of fuzzy logic for easily encoding the heuristics, plays an important role in implementing truly intelligent controllers. 
In complex processes, the relationships between the process parameter set-points and the resultant product quality are poorly understood; there is inadequate knowledge on how to adjust the process set-points to optimize a desired product quality. For example, in the automobile painting process, set-points must be determined for parameters such as booth temperature, humidity, vehicle movement speed, paint flow rate, plume-shaping air flow rate, downdraft ventilation air flow rate, and oven bake temperature and duration. Because the relationships between these process parameters and the resultant product quality (e.g., paint gloss and uniformity) are poorly understood, the parameter set-points are usually determined based on experience and trial-and-error. Great payoff in improved product quality and process efficiency can be gained by developing control systems that can automatically determine the optimal set-points and adjust the set-points to adapt to changing process conditions.

Various methods developed for learning fuzzy models from data (e.g., Jang 1993; Chiu 1994) can form the basis of such a system. A fuzzy model learned from process data provides an estimation of the full response surface for the process (i.e., estimates the full functional relationship between set-point values and product quality measures). A search algorithm, such as gradient descent, can then be applied to the estimated response surface to obtain an estimate of the optimal setpoint values. A simple example is shown in Figure 5, where a fuzzy model was learned from some data points that relate a set-point value $s$ to a quality measure $q$. The learned fuzzy model provides a continuous function $q=\mathrm{F}(s)$ that estimates the full response curve. Gradient descent search is then applied to $\mathrm{F}(s)$ to find the value of $s$ that minimizes $q$. In an actual process, $s$ is usually a high dimensional vector and $q$ is usually a weighted expression containing several quality parameters. For example, in wafer manufacturing, we may have [uniformity, stress $]=\mathrm{F}$ (pressure,temperature,gas_composition,...), and $q=w_{1}$ /uniformity + $w_{2}{ }^{*}$ stress, where $w_{1}$ and $w_{2}$ are weights.

After the set-point is changed to the estimated optimal value, actual quality measurements corresponding to the new set-point can be added as new data to refine the estimated response surface. Searching the refined response surface will produce a refined optimal set-point. In this way, the set-point can be iteratively optimized based on feedback.

Other modeling methods, such as polynomial and neural network models, can also be used for estimating the full response surface. The key motivation in using fuzzy models is that fuzzy rules are easy to understand, verify, and extend. Furthermore, the fuzzy rules can suggest good initial conditions for starting the search, whereas polynomial and neural network models provide no guidance for starting the search. 


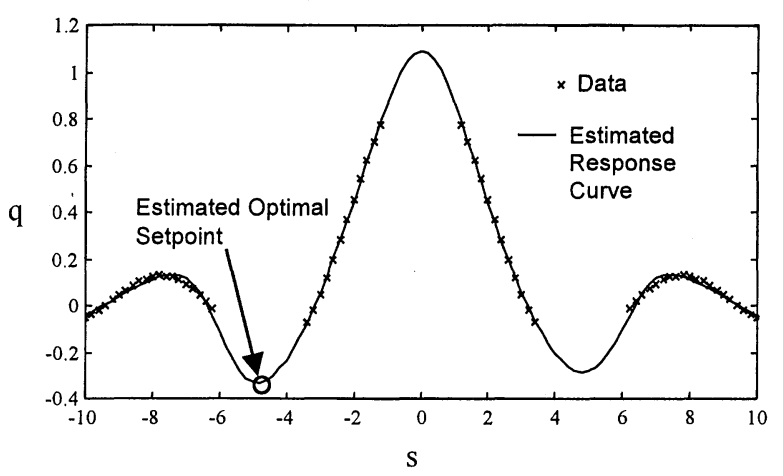

Figure 5 Fuzzy model learned from data provides an estimation of the full response curve. Gradient search is then applied to the estimated response curve to estimate the optimal set-point value.

\section{DEFINE PERFORMANCE METRICS}

How do we compare two system behaviors and judge which one is better? This issue arises when a control system designer must specify an optimality measure from which to search for the "best" control law or the "best" set-point values. The optimality measure defines the ideal system behavior and provides a quantitative measure of the closeness to the ideal behavior. Analytic optimality measures, such as the quadratic cost function, give the human designer a very limited language for expressing how to judge which system behavior is closer to the ideal. As an example, consider the common use of the root-mean-square (RMS) error as an optimality measure in system modeling. The ideal system behavior is one that produces zero error; however, in judging which system behavior is closer to the ideal, a designer may want to consider not only the RMS error, but also the maximum error, the average percentage error, and whether the errors occur on the conservative side. Complex trade-offs between these error types may be involved in selecting the "best" system behavior. The RMS error is only a rough approximation of how the human designer judges optimality.

The key point here is that a system considered optimal according to an analytic measure is not necessarily optimal according to human judgment. It is also important to keep in mind that analytic optimality measures are only mathematical tools for expressing what a designer wants a system to do; the true judge of optimality is the human designer, not the numerical value produced by the analytic measure. Unfortunately, we tend to choose an optimality measure based on whether it has nice mathematical properties, not based on whether it can express what is in the designer's mind.

The use of fuzzy logic to express optimality measures is one of the most valuable benefit that fuzzy logic brings to control applications. Fuzzy membership function 
is a natural framework for expressing the human designer's conception of the ideal system behavior and of how to measure closeness to the ideal behavior. Fuzzy logic can also provide smooth transitions in the optimality measure to emphasize certain control objectives as the operating condition changes.

The subway train control system developed by Hitachi (Yasunobu and Miyamoto 1985 ) is an example where fuzzy logic was applied to evaluate the optimality of control actions. For this particular system, the train's acceleration/deceleration is controlled by setting a power lever and a brake lever at different notch positions. Changing the notch position frequently or in large increments creates an uncomfortable ride. In addition to riding comfort, the controller must consider safety, on-time arrival, energy consumption, and stopping the train accurately at a specified position along the station platform. Optimizing train control requires trade-off between these multiple, often conflicting objectives.

The control method is based on predicting the outcome of each possible control action and then choosing the action that corresponds to the optimal outcome. A simple simulation of the train dynamics is used to predict the resultant speed, stopping position, and time of arrival for each possible choice of notch position. The optimality of each predicted outcome is then rated by a set of fuzzy rules, taking into account factors such as the degree to which the train is on schedule, the degree of safety of the predicted speed, closeness of the predicted stopping position to the desired stopping position, amount of notch change, and the elapsed time since the last notch change. Here fuzzy rules rate the different control outcomes by balancing multiple objectives in a way that reflects human's sensibility of "optimal". The notch position associated with the optimal outcome is then selected as the notch command.

In many control applications we know how we want a system to behave but find it difficult to express the desired behavior in an analytic formula. Fuzzy logic is a powerful tool for expressing human preferences and making the control system behavior accurately reflect these preferences.

\section{CONCLUSION}

There are many ways that fuzzy logic can be used in a control system to improve performance and reduce operating cost. The high payoff applications are usually not in replacing a conventional PID controller with a fuzzy PID controller, but in using fuzzy logic at higher levels of control. The previous application examples illustrate the wide range of opportunities that exist and the many different ways that fuzzy logic can be used to complement conventional controllers. A designer who wishes to exploit the full potential of fuzzy logic must maintain a broad view of the different aspects of a control problem and be creative in applying fuzzy logic where appropriate. 


\section{REFERENCES}

Bonissone, P.P., Badami, V., Chiang, K.H., Khedkar, P.S., Marcelle, K.W., and Schutten, M.J. (1995) Industrial applications of fuzzy logic at General Electric, Proceedings of the IEEE, 83, 450-465.

Chand, S. (1991) On-line, self-monitoring tuner for proportional integral derivative controllers, Proc. 30th IEEE Conf. on Decision and Control, Brighton, England.

Chiu, S. (1994) Fuzzy model identification based on cluster estimation, Journal of Intelligent and Fuzzy Systems, 2, 267-278.

Froese, T., von Altrock, C., and Franke, S., (1994) Optimization of a watertreatment system with fuzzy logic control, Proc. $3^{\text {rd }}$ IEEE Int'l Conf. on Fuzzy Systems, Orlando, USA, 1614-1619.

Gonsalves, P. and Zacharias, G. (1994) Fuzzy logic gain scheduling for flight control, Proc. $3^{\text {rd }}$ IEEE Int'l Conf. on Fuzzy Systems, Orlando, USA, 952-957.

Jang, J.S.R. (1993) ANFIS: Adaptive-network-based fuzzy inference system. IEEE Trans. Syst., Man, Cybern., 23, 665-685.

Sugeno, M. and Kang, G.T. (1986) Fuzzy modeling and control of multilayer incinerator, Fuzzy Sets and Systems, 18, 329-345.

Takahashi, H. (1991) A method for predicting the driving environment using fuzzy reasoning, Proc. IEEE Round Table Discussion on Fuzzy and Neural Systems, and Vehicle Applications, Tokyo, Japan.

Yasunobu, S. and Miyamoto, S. (1985) Automatic train operation system by predictive fuzzy control, in Industrial Applications of Fuzzy Control, ed. M. Sugeno, North-Holland, Amsterdam.

Yokogawa (1990) Yokogawa UT15/UT14 Digital Indicating Controllers, technical information publication \# TI 5B4A7-01E, Yokogawa Electric, Tokyo, Japan.

\section{BIOGRAPHY}

Stephen Chiu received the double B.S. degree in mechanical engineering and nuclear engineering from the University of California at Berkeley in 1983 and the S.M. degree in mechanical engineering from the Massachusetts Institute of Technology in 1985. He joined Rockwell's Science Center in 1985, where he is currently a Research Scientist. His research interests are in fuzzy and neuro-fuzzy systems for control, modeling and pattern classification. 